\title{
Does the Coordination of Verbal and Motor Information Explain the Development of Counting in Children?
}

\author{
Valérie Camos, Pierre Barrouillet, and Michel Fayol
}

\author{
L.E.A.D., Université de Bourgogne, Dijon, France
}

\begin{abstract}
Counting is often considered to be the coordination of two actions: saying the numberwords and pointing to each object. We report three experiments to test the hypothesis that this coordination requires the use of the central executive (A. D. Baddeley, 1990), and that the cost of coordination decreases with age. Participants were 5- and 9-year-old children and adults. At all ages tested, the manipulation of the difficulty of each component affected counting performance but did not make coordination more difficult. These results suggest that, at least from the age 5 , counting is a procedure in which the control of coordination is not attention demanding. ๑2001 Academic Press
\end{abstract}

Key Words: counting; coordination; cognitive development; working memory.

Piaget and Szeminska (1941) conceived of the concept of number as a synthesis of inclusion and seriation: children would acquire it at around age 6 when they achieved the conservation of discrete quantities. From this perspective, the use of numbers before this age could be no more than a verbal facet of the construction of the concept. Nevertheless, Gréco (1962) emphasized the role of counting in the acquisition of the conservation of quantity, and subsequent studies (Fuson, 1988; Gelman, 1983; Gelman \& Gallistel, 1978) have considered the processes of quantification, especially counting, as precursors of arithmetic.

Apart from Fuson's (1988) work, few studies have been dedicated to counting performance and the functional constraints which hinder it. The counting of arrays requires (1) saying number-words in the correct order, and (2) visually or manually pointing to each object once and only once (Beckwith \& Restle, 1966; Potter \& Levy, 1968). The coordination of these two components makes it possible to establish a strict correspondence between objects and number-words while avoiding double-counting or omissions (Fayol, 1985, 1990; Fuson, 1988).

We thank the children, N. Misset, and G. Valentin for the friendly welcome to their school and J. Boisselet, M. Buffet, and I. Niveau for help in testing participants. We thank J. Bisanz, J. N. Towse, and an anonymous reviewer for their helpful comments on the first draft of the manuscript. We also thank R. Engle for careful reading.

Address correspondence and reprint requests to Valérie Camos or Pierre Barrouillet, LEAD, Université de Bourgogne, Faculté des Sciences, 6, Boulevard Gabriel, 21000 Dijon, France. E-mail: camos@psycho.univ-paris5.fr or barouil@u-bourgogne.fr. 
Several recent models imply that the simultaneous mobilization of the two components and their coordination is attention demanding and has an impact on performance (Baddeley, 1990; Case, 1985). These models seem well suited to understanding complex activities such as counting, and constitute a good framework for the analysis of developmental phenomena. The aim of the research described here was to test the hypothesis that the coordination of the two components of counting has a cognitive cost and, most importantly, that the developmental improvement in counting stems from a developmental decrease in the cognitive cost of coordination.

Performance in counting should depend on the cost of both saying words and pointing at objects. Speed and accuracy of saying the number words depend on retrieving them in the correct order ("un" to "seize," one to sixteen; Fuson, Richards, \& Briars, 1982; Miller, 1996) and elaborating them ("dix-sept," "dixhuit," . . . . seventeen, eighteen . . .) by combinatory rules ("trente-cinq," thirtyfive). Dehaene and Mehler (1992) have shown large number-words are used less frequently, and thus their retrieval should be more difficult than that of small number-words. Moreover, saying large numbers takes longer because they often have more syllables (e.g., one, two vs seventeen, twenty-one). As long as retrieval and elaboration are not yet automatized, they have a cognitive cost, which leads to greater access time and more errors. Indeed, Nairne and Healy (1983) found such results when their adult participants counted backwards. Thus, the cost of saying number words depends on the size of the arrays to be counted and on the verbal chain used.

Speed and accuracy of the pointing component depend on both the size and the arrangement of the arrays (Shannon, 1978). Gelman and Meck (1983) showed that increasing the difficulty of pointing hurts counting performance. Regularly arranged objects allow systematic visual scanning of the array and thus decrease the number of errors (Aoki, 1977; Beckwith \& Restle, 1966; Newman, Friedman, \& Gockley, 1987; Potter \& Levy, 1968). Any factors improving the discrimination of the objects facilitate pointing. For example, displaying objects in different colors facilitates the distinction between counted and uncounted objects, and improves performance (Frick, 1987; Schaeffer, Eggleston, \& Scott, 1974; Towse \& Hitch, 1996; Trick \& Pylyshyn, 1994).

Even with successful performance of the two components of counting, pointing and saying, ultimately they must be coordinated. Their unsuccessful coordination causes counting errors. The cost of this coordination depends on the difficulty in saying and in pointing (Briars \& Siegler, 1984). Wilkinson (1984) had already predicted that the integration of pointing and saying would be all the more difficult the higher the cost of pointing is. He observed an increasing variability in counting performance as the complexity of pointing increased, and concluded that coordination constrains the success of counting. He also ascertained that ontogenetic development improves the coordination of the two initially independent components. However, Wilkinson (1984) did not propose any hypothesis about the mechanisms involved in the integration of the two components. 
Miller and Stigler (1987) analyzed this integration, and, considering the limits of cognitive resources, predicted that the difficulty of pointing would have a weaker effect when saying the numbers is less demanding. They compared Chinese children whose language makes transparent the combinatory rules (Miura et al., 1994) to American children (nontransparent rules). Contrary to the hypothesis, the language used and the difficulty of pointing affected performance, but there was no interaction meaning that the two processes run without interference. The authors concluded that the coordination of the two components is a modular activity, in contradiction to Wilkinson's (1984) conclusions.

To summarize, it is clear that the increase in the difficulty of each component (pointing and saying) has an impact on counting performance. However, the data concerning the possible cost of coordination are contradictory. Sometimes coordination is considered to have a cognitive cost (Wilkinson, 1984), and sometimes not (Miller \& Stigler, 1987).

Towse and Hitch (1997) studied the coordination of the two components within the framework of Baddeley's working memory model (Baddeley, 1990; Baddeley \& Hitch, 1974). Within this perspective, each component depends on a specific subsystem of working memory, i.e., saying on the phonological loop (Logie \& Baddeley, 1987), pointing on the visual sketchpad, but their coordination is assumed by a limited-capacity attention mechanism called the central executive. Thus, an increase in the cognitive cost of each component should lead to a more demanding coordination, and these effects should interact. Subsequently, 7- to 9-year-old children were subjected to a series of experiments simultaneously manipulating the difficulty of the verbal and the motor activity. However, as previously observed by Miller and Stigler (1987), these effects were additive. This result suggests that integrating the two activities mobilizes few resources of the central executive and has no cost. According to Towse and Hitch (1997), such results could nevertheless be interpreted within the framework of Baddeley's model. For example, sufficient control of each working memory subsystem (i.e., the phonological loop and the visual sketchpad) could make the intervention of a central system unnecessary. Alternatively, coordination could be only an emergent property of the interaction between the various processes involved, a fact which characterizes integrated and frequently practiced activities.

Although the results of Miller and Stigler (1987) and Towse and Hitch's (1997) suggest that coordination does not have any cognitive cost, the evidence is indirect. Even if some authors have questioned the theoretical framework of resources (Navon, 1984) or suggested that such interaction could result from output interference (Brainerd \& Reyna, 1989), the lack of interaction does not force the conclusion that coordination has no cost and that the two tasks do not compete for common resources. Towse and Hitch (1997) have suggested that each component (i.e., visual pointing and saying) could have so small a cost that the predicted interaction would not appear; that is, the task is insensitive to the interaction. This suggestion fits with an observation by Anderson, Reder, and Lebière (1986), who studied arithmetic problem-solving and the concurrent maintenance of information in short- 
term memory. While Carlson and Sulivan (1989) failed to find any interaction between the difficulty of problems and the number of items to be maintained in memory, Anderson et al. (1986) found the interaction when the difficulty of the problems and the number of items were increased. This suggests that the interaction appears only when the two tasks induce a cognitive overload. Towse and Hitch (1997) may have failed to find the predicted interaction because the components of counting are automatized in children having already long experience with counting.

Consequently, the "search-for-interaction" paradigm is perhaps not the most appropriate to test the hypothesis of a demanding coordination in counting. The following experiments aimed to overcome the difficulties raised by Towse and Hitch's paradigm. We used a paradigm which joined the componential approach used by Wilkinson (1984) with the factorial design favored by Towse and Hitch (1997). Our experiments were designed to evaluate the time required to execute each component separately (i.e., pointing and saying) and to compare these times with the counting time among the same participants. Similar comparisons were planned on the errors. The difficulty of pointing was evaluated by measuring the errors and the time required to manually point at objects which would be counted afterward. The same measurements could be used for saying alone, because the number of errors and the maximum speed reached by a participant reflect the level of automatization of saying the numbers. Following Towse and Hitch's hypothesis, any increase in the difficulty of one of the components should increase the cost of coordination. This should result in greater differences between counting time (or error rate) on the one hand, and pointing and saying times (and error rates) on the other hand. Moreover, these differences should decrease with development.

The hypothesis of a demanding coordination of pointing and saying implies that overall counting time must be longer than the time required by the slowest of the two components: $C>\operatorname{Max}[P, S]$, where $C, P$, and $S$ refer to the time needed by counting, pointing alone, and saying alone, respectively. The same argument applies to the probabilities of errors. If the two components of counting are independent, that is, without interference, the probability of counting errors $p(C)$ is given by the equation $p(C)=p(S)+p(P)-p(S) \times p(P)$, where $p(S)$ is the probability of errors in saying alone and $p(P)$ the probability of errors in pointing alone. In contrast, a probability $p(C)$ greater than $p(S)+p(P)-p(S) \times p(P)$ might indicate an additional cost due to the demanding coordination of the two components.

The first experiment compared the times and errors observed for 5- and 8-yearold children and adults to count small (11 to 18 dots) and large (24 to 36) arrays with the times and errors for (a) saying number-words and (b) pointing to objects in similar arrays. The hypothesis of a demanding coordination predicts that, on the one hand, $C>\operatorname{Max}[P, S]$, and, on the other, $p(C)>p(S)+p(P)-p(S) \times p(P)$. These differences should be all higher with younger subjects, assuming that young children have lower processing capacities than older children and adults. Moreover, these differences should grow as the cost of one or other component increases. Thus, Experiments $2 \mathrm{a}, 2 \mathrm{~b}$, and 3 are intended to replicate the previous 
comparisons by manipulating the cognitive load of one component (saying or pointing). Experiments $2 \mathrm{a}$ and $2 \mathrm{~b}$ manipulated the cost of saying. In Experiment $2 a$, this cost could be very low (say "ba" instead of each number-word) or high, using the alphabet, a less automatized chain than the numberline, especially for the youngest children. Experiment $2 \mathrm{~b}$ replicated the paradigm of the previous experiment, using three differently automatized numberlines for French native speakers: French, English, and Tahitian. Experiment 3 manipulated the cost of counting by making the pointing of targets more difficult. Six- and 9-year-old children and adults had to count targets which differed from the distracters in their color alone or by their color and shape, the conjunction of two features making the search process more costly (Treisman \& Gelade, 1980).

\section{EXPERIMENT 1}

The hypothesis of a demanding coordination of pointing and saying predicts that the time needed to count an array would be greater than the time required to execute the slowest component. This difference would decrease with age because experience could lead to an automatization of each component and development would lead to an increase in cognitive resources. These two changes would facilitate the integration of the components by diminishing their relative cost. On the one hand, the automatization decreases the resources necessary for the fulfillment of each separate component (Barrouillet \& Fayol, 1998; Case, 1985; Logan \& Klapp, 1991). On the other, the increase in resources (Halford, 1993; PascualLeone, 1988) facilitates the integration of the two components, and makes an overload less probable. Because pointing and saying would be automatized more rapidly for the small arrays (due to the less demanding control of pointing and the earlier learning of the beginning of the numberline), the cost of coordination would be lower for the small arrays than for the larger ones. Thus, the difference between counting time $(C)$ and the time of the slowest of the two components (Max $[P, S]$ ) would increase with the size of the array, and would be much greater for the younger children. The same hypothesis applies to the probability of error.

\section{Method}

Participants. Twenty-six children in the last year of nursery school (14 female and 12 male, mean age $=5 ; 3$ years, $S D=4$ months), and 25 third-graders ( 9 female and 16 male, mean age $=8 ; 5$ years, $S D=5$ months) participated. All were French native speakers and attended upper-middle-class public schools. The adults participants were 31 undergraduate psychology students (29 female and 2 male, mean age $=21 ; 2$ years, $S D=20$ months) at the Université de Bourgogne.

Material. Black round stickers (dots) of 16-mm diameter were stuck in nonsystematic arrangements on $24 \times 32-\mathrm{cm}$ sheets for the small arrays (size 1:11 to 18 dots) and on $24 \times 65-\mathrm{cm}$ sheets for the large arrays (size 2:24 to 36). Sixteen arrays ( 8 for both sizes) were constructed for the pointing task and 16 others for the counting task, in order to avoid any training effect between the two tasks. Two examples (10 and 12 dots) and 8 training arrays ( 7 to 12$)$ were constructed. 
Procedure. The participants performed three tasks individually. In the first, pointing, they were asked to point at each dot using a finger, as fast as possible without making any mistakes (omission or repetition). After two examples performed by the experimenter, the participants were asked to point in 8 training arrays during which the experimenter corrected errors. The 16 experimental arrays that followed were presented in a different random order for each participant. The experimenter noted on-line the total time of pointing and the number and type of errors (omissions or double-pointing).

In the second task, saying, participants were audiotaped saying the numeric chain aloud from 0 to 40, one by one, as fast as possible but clearly. After one practice trial during which the experimenter corrected any errors, they performed the task three times. The times required to say the numbers from 0 to 18 on the one hand, and from 0 to 36 on the other, were measured for each of the three trials from the recording, and averaged as the measure of the two saying times (0-18 and 0-36). For participants able to count to 36, the number of errors was also recorded from the audiotaped recorded.

In the third task, counting, participants were asked to count dots aloud, as fast and accurately as possible, while pointing at them one by one. After two examples performed by the experimenter, participants were given 8 training arrays. If an error occurred, the experimenter repeated the instructions. The 16 experimental arrays were then presented in a random order that differed for each participant and that differed from the order used in the pointing task. For each array, the experimenter recorded number and type of errors of pointing during the session, while errors of saying and total counting times were taken from an audiotape.

The order of the three tasks was counterbalanced between participants who were randomly assigned to one of the six possible orders. For children only, these three tasks were performed during three different sessions (one task per session) held on successive days.

\section{Results}

The error rate was the percentage of trials in which at least one error occurred for each task and size. Dependent measures of times were (a) the mean pointing time per dot averaged for each size (total pointing time/number of dots pointed at), (b) the mean saying time per word averaged for each size (total saying time/number of number-words said), and (c) the mean counting time per dot averaged for each size (total counting time/number of dots counted). The time analyses were performed for the successful trials only, thus retaining 31 adults, 24 8year-old and 235 -year-old children, but only 105 -year-old children for the large size arrays. Unless otherwise noted, all statistical tests reported were significant at $p<.05$.

The mean times and error rates for each task were analyzed with an analysis of variance (ANOVA) in which Age was a between-subject factor and Size was a within-subject factor. Young children were slower and made more errors than older children and adults. This was reflected by a significant main effect of age 
on times, $F(2,61)=284.78, p<.001, M s_{\mathrm{e}}=.004, F(2,51)=50.18, p<.001$, $M S_{\mathrm{e}}=.019, F(2,53)=65.95, p<.001, M S_{\mathrm{e}}=.014$, and errors, $F(2,64)=46.66$, $p<.001, M S_{\mathrm{e}}=.056, F(2,64)=28.38, p<.001, M S_{\mathrm{e}}=.093, F(2,64)=37.87, p<$ $.001, M S_{\mathrm{e}}=.084$, for the three tasks, pointing, saying, and counting, respectively. Large arrays resulted in longer pointing and counting time per dot than small arrays, $F(1,61)=85.34, p<.001, M S_{\mathrm{e}}=.0004, F(1,53)=26.81, p<.001, M S_{\mathrm{e}}=$ .003 respectively, and greater saying times per word, $F(1,51)=280.84, p<.001$, $M S_{\mathrm{e}}=.0014$. Large arrays resulted also in more errors on each of the three tasks, pointing, saying and counting, $F(1,64)=90.10, p<.001, M S_{\mathrm{e}}=.018, F(1,64)=$ $33.22, p<.001, M S_{\mathrm{e}}=.027, F(1,64)=78.28, p<.001, M S_{\mathrm{e}}=.028$, respectively. The size effect was more pronounced for younger children on times and errors (Table 1). This Size $\times$ Age interaction was significant on errors and times ( $p$ s < .003 ), except for counting times for which the interaction did not reach significance, $p=.07$.

Comparison between counting time and Max $[P, S]$ time. To compare $C$ and Max $[P, S]$, ANOVAs with Order (6) as between-subject factor and Time (2: $C$ vs Max $[P, S])$ as within-subject factor were performed separately for each age and size. This procedure was adopted because (a) the variances differ with age and size, and (b) only 10 5-year-old children achieved at least one correct response in each task for the large size.

The effect of order was not significant and did not interact with Time. Contrary to the costly coordination hypothesis, counting time in 5-year-old children was always lower than the time needed by the slowest of the two components (Max

TABLE 1

Mean Times (in ms) and Percentage of Errors (in Parentheses) in Experiment 1

\begin{tabular}{|c|c|c|c|c|c|c|}
\hline \multirow[b]{3}{*}{ Set size: } & \multicolumn{6}{|c|}{ Age } \\
\hline & \multicolumn{2}{|c|}{6} & \multicolumn{2}{|c|}{8} & \multicolumn{2}{|c|}{ Adults } \\
\hline & Small & Large & Small & Large & Small & Large \\
\hline \multicolumn{7}{|l|}{ Tasks } \\
\hline \multirow[t]{2}{*}{ Saying } & 549 & 607 & 200 & 291 & 166 & 235 \\
\hline & (19) & (64) & (3) & (4) & (1) & (1) \\
\hline \multirow[t]{2}{*}{ Pointing } & 489 & 526 & 307 & 340 & 198 & 212 \\
\hline & (39) & (69) & (30) & (46) & (7) & (17) \\
\hline Max. time & 629 & 670 & 309 & 344 & 199 & 239 \\
\hline Predicted errors & $(50)$ & (87) & (26) & (47) & (7) & (16) \\
\hline \multirow[t]{2}{*}{ Counting } & 539 & 601 & 305 & 346 & 215 & 241 \\
\hline & (46) & (85) & (37) & (52) & (10) & (25) \\
\hline
\end{tabular}

Note. Max time is the greater of the pointing and saying times. Mean Max time is the average of Max times obtained from each subject. Predicted errors is the percentage of errors in counting predicted from the independence of the two components, pointing and saying. The rates of errors in 8year-old children for small size when the Saying-Counting-Pointing order was discarded were 34 and $31 \%$ for counting and predicted, respectively. 
$[P, S]$ ). This difference was significant for the small arrays (539 vs $629 \mathrm{~ms}$ for counting and Max $[P, S]$, respectively), $F(1,17)=7.70, p=.013, M S_{\mathrm{e}}=.017$. Although the difference was in the same direction for large arrays (601 vs $670 \mathrm{~ms}$ for counting and Max, respectively), it failed to reach significance, due to the small number of children who had at least one correct trial in each of the three tasks $(n=10)$.

The difference between counting time and Max disappeared in 8-year-old children for both small (305 vs 309 ms for counting and Max, respectively) and large arrays (346 and $344 \mathrm{~ms}$ ), $p s>.20$. Amazingly, the predicted difference between Max and counting time appeared only in adults for the small arrays, where counting time (215 ms) was higher than the Max (199 ms), $F(1,25)=40.46, p<.0001$, $M S_{\mathrm{e}}=.0001$. There was no difference for the large arrays (241 vs $239 \mathrm{~ms}$ for counting and Max, respectively), $F(1,25)<1$.

Comparison between predicted and observed error rates in counting. The predicted error rate was given by the law of probability independence between pointing and saying, i.e., $p(C)=p(P)+p(S)-p(P) \times p(S)$. The error rates were analyzed with a design similar to that of the times analyses, except that Time was replaced by Rate (2: predicted vs observed).

As previously observed, the effect of Order was not significant, and did not interact with Rate except for the 8-year-old children in small size, $F(5,19)=3.70$, $p=.017, M S_{\mathrm{e}}=.03$. This interaction was only due to one specific order (Saying-Counting-Pointing) and disappeared when it was removed from the analysis, $F(4,16)=1.22, p=.34, M S_{\mathrm{e}}=.02$. Indeed, this order showed a totally reverse pattern of results compared with the five others.

Contrary to the costly coordination hypothesis, errors did not significantly differ from predicted rates at all age or size ( $p \mathrm{~s}>.11)$, except for the 8-year-old children in small size, $F(1,19)=5.09, p=.04, M S_{\mathrm{e}}=.032$. This difference was not significant when the order that elicited the previously discussed interaction was discarded, $F<1$. We also computed the analysis comparing the Time for the 8year-old children in small size without this order. No change occurred regarding the significance of the effects or the value of the different means.

To summarize, the hypothesis of a costly coordination of pointing and saying within counting was not supported by the pattern of results. First, the error rate observed in counting was not higher than the probability predicted by the independence between saying and pointing. Second, the results on times reflected the exact reverse of the expected developmental pattern. Indeed, counting took longer than the Max $[P, S]$ only in adults, but the two times did not differ in 8-year-old children and counting was even faster than the Max $[P, S]$ in the youngest children. Thus, this developmental pattern cannot be accounted for by decreased cost to coordinate saying and pointing.

\section{Discussion}

As predicted, the age and the size of arrays affected each component considered separately (pointing and saying) and had similar effects on counting times 
and errors. The youngest children counted slowly and made more errors than older children and adults. The counting of large arrays took longer and caused more errors than the counting of small arrays, this difference decreasing with age. Thus, these results supported the hypothesis that, for the youngest children, the two components of counting reflect a cognitive cost that decreases with age through the automation of processing and a developmental increase in cognitive capacities (Case, 1985; Halford, 1993; Pascual-Leone, 1988).

According to Baddeley's (1990) model, the coordination of two controlled activities should induce an additional cognitive cost. Thus, counting should take longer than the slower of saying and pointing $(\operatorname{Max}[P, S])$ and should result in more errors than the performance for each component could predict. In fact, the results contradicted these two predictions. The difference between counting time and Max $[P, S]$ did not decrease with age but increased, suggesting that there is no coordination cost in counting. This increasing difference cannot be due to a trade-off between speed and accuracy, because the error rate in counting never exceeded what the performance observed in pointing and saying could predict. Furthermore, the comparison between counting times and Max $[P, S]$ showed a compression effect in young children. This effect is akin to that described by Yee, Hunt, and Pelligrino (1991), who asked adults to coordinate a verbal and a visuospatial task. Nevertheless, our results are compatible with Miller and Stigler (1987) and Towse and Hitch (1997), who observed no interaction when the difficulty of each component was manipulated, contrary to what most of the resource models predict (Anderson et al., 1996; Baddeley, 1990; Turner \& Engle, 1989).

It was surprising that the counting time was lower than the Max in young children. A possible explanation could be that the cost of each component in the counting task was not sufficient to induce a costly coordination (Towse \& Hitch, 1997). Indeed, the effect of a costly coordination would appear only when the difficulty of the two components would lead to an overload (Anderson et al., 1996). Thus, an increase in the cognitive cost of one of the components could reveal a demanding coordination of verbal and motor processes during counting. To control for this possibility, the cognitive load of either saying or pointing was manipulated in Experiments 2a, 2b, and 3.

\section{EXPERIMENT 2A}

The aim of this experiment was to determine whether increasing the cost of saying would make its coordination with pointing more difficult. Participants were asked to count arrays using three types of verbal chain. The first and least demanding consisted of saying the syllable "ba" for each object pointed at (Baddeley, 1990). The second was the number line. The third was the alphabet, the less automatized and the more demanding in young children (Barrouillet \& Fayol, 1998). Any increase in the demand of saying would make its coordination with pointing more difficult. This should result in counting times greater than the time needed by the slowest component and in error frequencies higher than what can be predicted if pointing and saying are independent. These differences should 
be all the more prominent with less automatized verbal chains. As predicted in the previous experiment, these differences should decrease with age.

\section{Method}

Participants. Fifteen children in the last year of nursery school (10 female and 5 male, mean age $=5 ; 5$ years, $S D=3.3$ months), and 25 third-graders ( 16 female and 9 male, mean age $=8 ; 11$ years, $S D=6.6$ months) participated. All were French native speakers and attended upper-middle-class public schools. The adults were 32 students in psychology ( 29 female and 3 male, mean age $=22 ; 8$ years, $S D=47.8$ months) at the Université de Bourgogne.

Material and procedure. We used the same materials as in Experiment 1. Because younger children (mean age $=5 ; 5$ years) have a poor knowledge of the various verbal chains, only the small arrays were used (five arrays, 8 to 12 dots). The participants were asked to perform three tasks individually: pointing, saying, and counting, in that order. Indeed, in Experiment 1, the order had no effect either on times or error rates. Moreover, some young children experienced difficulties in pointing after counting because they were unable to inhibit the counting procedure previously activated. Thus, we chose an order in which pointing preceded counting to have a better measure of pointing speed. The Pointing-Saying-Counting order we used was the one that elicited the better chronometric performance for the three tasks without any noticeable increase in error rate in Experiment 1.

The procedure was similar to that of Experiment 1, except for saying and counting. Saying task was performed twice, except for 6-year-old children, who performed it once to shorten the experiment. The participants were recorded saying, as fast as possible, "ba - ba" for $10 \mathrm{~s}$, the number chain from 0 to 12 , and the alphabet. The counting task consisted of three conditions. The participants were asked to point at each dot while simultaneously saying "ba," to count the objects using the number chain and then the alphabet.

\section{Results}

For each task, we analyzed the number of errors and the times for the successful trials only, thus retaining 12 6-year-old children, 23 9-year-old children, and 29 adults. The analysis of each task revealed the same effects as previously observed. The older the participants were, the shorter the performance times and the lower the number of errors were for all tasks (Table 2).

To verify that verbal chains had different cost, we compared the three types of verbal chains (i.e., "ba," number line, and alphabet) for the saying and the counting tasks. A 3 (Age: 6-year olds, 9-year olds, and adults) $\times 2$ (Task: saying vs counting) $\times 3$ (Chain: "ba," numbers, and alphabet) ANOVA with repeated measures for the last two factors was conducted on times. The saying times $(267 \mathrm{~ms})$ were lower than the counting times $(362 \mathrm{~ms}), F(1,61)=141.30, p<.001, M S_{\mathrm{e}}=$ 5432. This effect interacted with age. The adults exhibited the smallest differences between the two tasks, $F(2,61)=3.25, p=.045, M S_{\mathrm{e}}=5432$. As predicted by our hypothesis, the use of the alphabet $(328 \mathrm{~ms})$ resulted in higher times 
TABLE 2

Mean times (ms) and Percentage of Errors (in Parentheses) in Experiment 2a

\begin{tabular}{|c|c|c|c|}
\hline & \multicolumn{3}{|c|}{ Age } \\
\hline & 6 & 9 & Adults \\
\hline Pointing & 537 (13) & 290 & $210 \quad(4)$ \\
\hline \multicolumn{4}{|l|}{ "ba... ba" } \\
\hline Saying & $381 \quad(0)$ & $197 \quad(0)$ & $188 \quad(0)$ \\
\hline Max time & $550(13)$ & 290 & 212 \\
\hline Counting & $515(31)$ & 313 & $240 \quad(5)$ \\
\hline \multicolumn{4}{|l|}{ Numbers } \\
\hline Saying & $440 \quad(0)$ & $197 \quad(0)$ & $163(0)$ \\
\hline Max time & $568(13)$ & 290 & 212 (4) \\
\hline Counting & $515(35)$ & 307 (14) & 235 (11) \\
\hline \multicolumn{4}{|l|}{ Alphabet } \\
\hline Saying & $480(40)$ & $201 \quad(8)$ & 152 (8) \\
\hline Max time & $586(47)$ & $292 \quad$ (9) & $210(12)$ \\
\hline Counting & $556(40)$ & 327 (14) & 252 (6) \\
\hline
\end{tabular}

Note. For Max time see footnote in Table 1. In accompanying parentheses we indicate the predicted errors, that is, the percentage of counting errors predicted from the independence of the two components, pointing and saying.

than the other two chains ("ba": $306 \mathrm{~ms}$; numbers: $309 \mathrm{~ms}), F(2,122)=9.64$, $p<.001, M S_{\mathrm{e}}=1666$. This effect interacted with age, $F(4,122)=8.84, p=<.001$, $M S_{\mathrm{e}}=1666$, but not with the tasks, $p=.27$. In fact, the Age $\times$ Chain interaction was significant only in the saying task, $F(4,122)=10.87, p<.001, M S_{\mathrm{e}}=1865$, but not for the counting task, $F<1$. Then, the Age $\times$ Chain $\times$ Task interaction was significant, $F(4,122)=4.17, p<.01, M S_{\mathrm{e}}=1598$.

A second ANOVA was performed on the number of errors using the same design as in the previous analysis. This confirmed the results of the analysis of the times. Errors were less frequent for saying (5\%) than for counting (14\%), $F(1,69)=28.38, p<.0001, M S_{\mathrm{e}}=.05$. The use of the alphabet induced more errors (15\%) than the other two chains (5 and 9\%, respectively, for "ba" and numbers), $F(1,69)=22.2, p<.0001, M S_{\mathrm{e}}=.05$.

A 2 (Time: counting vs Max $[P, S]$ ) ANOVA with repeated measures was performed for each age group and chain. As in Experiment 1, the developmental pattern of the differences between the counting time and the Max $[P, S]$ contradicted the hypothesis that the coordination between pointing and saying is less and less demanding with age. The counting times were significantly greater than the Max $[P, S]$ in adults and in 9-year-olds for the three chains (i.e., "ba," numbers, and alphabet), $p$ s $<.02$, but always smaller than the Max $[P, S]$ in 6-year-olds. This latter difference did not reach significance for the "ba" and the numeric chain, $p \mathrm{~s}>.11$, and just failed to reach significance for the alphabet, $F(1,11)=$ $4.27, p=.06, M S_{\mathrm{e}}=15,560$. 
The prediction that the difference between counting time and Max $[P, S]$ should be larger with the less automatized chains was tested using a 2 (Time: counting vs Max [P, S]) $\times 3$ (Chain: "ba," numbers, alphabet) ANOVA with repeated measures on the two factors in each age group. Contrary to the costly coordination hypothesis, there was no interaction between Chain and Time in 6-year-old children $(F<1)$ and in adults, $F(2,56)=1.22, p>.05, M S_{\mathrm{e}}=1116$. Thus, although counting took longer with the alphabet than with the other chains, suggesting that it involves an higher cognitive load, the difference between counting and Max $[P, S]$ remained unchanged across the verbal chains. Counting with the alphabet resulted in a higher difference between counting and Max $[P, S]$ in 9-year-old children only, $F(2,44)=4.34, p<.02, M S_{\mathrm{e}}=226$. Although this interaction was in line with the hypothesis of a costly coordination, the overall pattern of results did not fit with this hypothesis, because the predicted interaction did not appear with the youngest children, who, once more, showed counting time smaller than the time for the slowest component.

When errors are considered, the hypothesis of a demanding coordination predicted that $p(C)$ would be greater than $p(P)+p(S)-p(P) \times p(S)$. This effect would decrease with age and with the automation of the verbal chain. A one-way ANOVA that compared observed and predicted rates of errors was conducted for each age and chain. The observed and predicted rates of errors in counting did not differ significantly in adults and in 9-year-olds, $p$ s $>.13$, except for the numeric chain in 9year-olds for whom the observed rate was higher than the predicted rate, $F(1,24)$ $=6.51, p<.05, M S_{\mathrm{e}}=.031$. Similarly, for the younger children, the observed rate of errors was significantly greater than the predicted rate for the "ba" chain, $F(1$, $14)=8.89, p<.01, M S_{\mathrm{e}}=.025$, and the numeric chain, $F(1,14)=8.26, p=.012$, $M S_{\mathrm{e}}=.04$. More interestingly, these two rates did not differ significantly for the alphabet, $F(1,14)<1$. The interaction between rates and chains did not reach significance for both 6-year-old and 9-year-old children, $F(2,28)=2.86, p>.05, M S_{\mathrm{e}}=$ .06 , and $F(2,48)=2.22, p>.05, M S_{\mathrm{e}}=.02$, and was significant for the adults only, $F(2,62)=3.25, p<.05, M S_{\mathrm{e}}=.02$. However, the trend of this interaction was reverse as predicted because counting elicited more errors than predicted when "ba" and number chains were used but fewer errors with the alphabet.

\section{Discussion}

This experiment tested the hypothesis that saying the alphabet is more demanding than that of a number chain, which is in turn more demanding than the repeated pronunciation of a single syllable ("ba"). Both the times and error rates in counting and saying confirmed this hypothesis. Counting with the alphabet took longer than with the number line, with identical rates of error (mean of $20 \%$ in both conditions). However, the difference between the counting time and the time required to perform the slowest component was affected only slightly by the verbal chain used. This result, which is incompatible with the hypothesis of a demanding coordination of the two counting components, was corroborated by the analyses on errors. Indeed, the difference between 
observed and predicted rates of errors was smaller with the alphabet than with the other chains.

Experiment 2a was based on the hypothesis that alphabetic counting is more demanding than numeric counting. While the use of the alphabet clearly induced longer counting times, it did not induce a greater number of errors of counting. Thus, it is possible that saying the alphabet was not sufficiently demanding to increase the cost of coordination between pointing and saying. Consequently, we designed a new experiment in which adults counted in their native language (French), in a welllearned foreign language (English), and in a recently learned language (Tahitian).

\section{EXPERIMENT 2B}

The participants counted using three verbal number lines belonging to different languages: French, English, and Tahitian. Since French was the mother tongue of all the participants, reciting the number line in this language was less demanding, especially for the adults. In contrast, this skill was less highly automatized for English, even though all the participants had received at least 5 years training in English. Finally, the production of a new chain in Tahitian was expected to induce a greater cost than the other chains. As in Experiment 2a, the use of less automatized chains should increase the counting time and the number of errors, as well as the difficulty of coordinating saying with pointing. This difficulty should induce counting times greater than times needed to perform the slowest component (Max $[P, S]$ ), and error probabilities greater than those predicted on the basis of performance in pointing and saying.

\section{Method}

Participants. Twenty-eight adult psychology students at the Université de Bourgogne ( 25 female and 3 male, mean age $=20 ; 0$ years, $S D=22$ months) took part in the experiment. All of them were French native speakers and had studied English for at least 5 years.

Material and procedure. The material was similar to that of Experiment 1. Small size was represented by six arrays (8 to 13 dots) as was the large size (18 to 23 dots). The participants attended a 20-min training sessions on each of the 4 days preceding the experiment to learn the Tahitian number line. Their knowledge was tested at the beginning of the experiment. The procedure was similar to that of Experiment 2a. The participants were asked to perform three tasks individually: pointing, saying, and counting. During the saying task, participants counted from 1 to 30 successively in the three languages. The saying times from 0 to 13 and from 0 to 23 were noted as the number of errors. Participants performed this task twice. Likewise, in the counting task, each participant used the three number lines in succession. The sequence of the three languages was counterbalanced between subjects.

\section{Results}

We performed ANOVAs on times and errors for the pointing task, in which the Size (2: large vs small) was a within-subject factor, and for the saying and count- 
ing tasks in which the Size and the Chain (3: French, English, and Tahitian) were within-subject factors. The times analyses were performed on data from participants who had at least one correct trial per condition (i.e., 15 of 28 participants).

As in the previous experiments, counting large arrays induced more errors and took longer for all three tasks. As we expected, saying and counting in French were faster (216 and $233 \mathrm{~ms}$, respectively) than in English (353 and $319 \mathrm{~ms}$ ) or Tahitian (743 and $655 \mathrm{~ms}$ ): saying, $F(2,28)=60.18, p<.001, M S_{\mathrm{e}}=37,265$; counting, $F(2,28)=124.78, p<.001, M S_{\mathrm{e}}=11,943$. The Size effect increased the less automatized the chain was. This interaction was significant both for saying, $F(2,28)=26.97, p<.001, M S_{\mathrm{e}}=6209$, and for counting, $F(2,28)=73.94$, $p<.001, M S_{\mathrm{e}}=2365$. The same effects were observed on errors. The errors were more frequent, the more recently the language was learned $(0,26$, and $47 \%$ in saying in French, English, and Tahitian, 8, 19, and 50\%, respectively in counting), $F(2,54)=31.1, p<.001, M S_{\mathrm{e}}=.10$ in saying, $F(2,54)=97.9, p<.001, M S_{\mathrm{e}}$ $=.06$, in counting. Similarly, the increase in the size of the arrays induced an increase in the number of errors which was all the greater the less familiar the participants were with the language used (see Table 3), $F(2,54)=8.8, p<.001, M S_{\mathrm{e}}$ $=.03$, in saying, $F(2,54)=14.6, p<.001, M S_{\mathrm{e}}=.04$, in counting. Thus, counting in English or in Tahitian was much more difficult than counting in French.

A 2 (Times: $C$ vs Max) $\times 3$ (Chain: French, English, and Tahitian) $\times 2$ (Size: small vs large) ANOVA revealed that the mean counting time (402 ms) was lower than the mean Max (446 ms), $F(1,14)=5.32, p=.04, M S_{\mathrm{e}}=16,155$. This difference appeared to be greater the less automatized the employed language was (for $C$ and Max, respectively, 233 and $239 \mathrm{~ms}$ in French, 319 and $355 \mathrm{~ms}$ in English, 655 and $743 \mathrm{~ms}$ in Tahitian) but the interaction between times and chains was not significant $(p=.22)$. Moreover, there was no significant difference between observed (26\%) and predicted (27\%) rates of errors in counting, $F<1$.

TABLE 3

Mean times (ms) and Percentage of Errors (in Parentheses) in Experiment $2 b$ (in Adults Only)

Language used for counting

French

English

Tahitian

\begin{tabular}{lrrrrrr}
\hline Set size: & Small & Large & Small & Large & Small & Large \\
\hline Tasks & & & & & & \\
Saying & 199 & 233 & 311 & 394 & 586 & 900 \\
& $(0)$ & $(0)$ & $(14)$ & $(38)$ & $(34)$ & $(61)$ \\
Pointing & 212 & 237 & 212 & 237 & 212 & 237 \\
& $(2)$ & $(6)$ & $(2)$ & $(6)$ & $(2)$ & $(6)$ \\
$\quad$ Max. time & 226 & 253 & 314 & 396 & 586 & 900 \\
$\quad$ Predicted errors & $(2)$ & $(6)$ & $(16)$ & $(43)$ & $(36)$ & $(63)$ \\
Counting & 217 & 249 & 249 & 388 & 488 & 821 \\
& $(5)$ & $(11)$ & $(7)$ & $(30)$ & $(33)$ & $(68)$ \\
& & & & & &
\end{tabular}

Note. For Max time and Predicted errors see footnote in Table 1. 
To summarize, when participants counted with number lines which were either largely (i.e., English) or very largely nonautomatized (i.e., Tahitian), the counting times were lower than the Max $[P, S]$. This effect echoes the facilitation effect observed in younger children in Experiment 1 and will be discussed later. The observed error rates were not greater than the predicted rates. No manipulations of the cost of saying the verbal chain had the effects predicted by the hypothesis of a demanding coordination of pointing and saying. The use of largely nonautomatized chains increased the number of errors, but never over the value predicted by the independence of pointing and saying. These chains increased the saying and counting times, but did not affect the difference between counting and $\operatorname{Max}[P, S]$.

Nevertheless, counting times, although frequently shorter than Max (see Tables 1-3), were rarely shorter than pointing times. When this occurred, error rates were correspondingly higher (i.e., conditions "ba" and numeric at age 6, Experiment 2a). The time required for pointing therefore strongly constrained the counting time. The aim of the following experiment was to verify that an increase in the load of pointing could induce an increase in counting times as well as in the difficulty of coordinating pointing and saying.

\section{EXPERIMENT 3}

In this experiment, the participants counted targets that differed from the distracters on one (color) or two features (color and shape). When the search for targets requires the conjunction of two features, the search process is more demanding (Treisman \& Gelade, 1980). As coordination is more difficult when the components of counting are less automatized, and therefore more demanding, we would expect the cost of coordination to be greater when the detection of targets requires the conjunction of the object's color and shape.

\section{Method}

Participants. Twenty-five first-graders ( 13 female and 12 male, mean age $=6 ; 3$ years, $S D=3.5$ months), and 30 third-graders (10 female and 20 male, mean age $=$ $8 ; 8$ years, $S D=7.0$ months) participated. All were French native speakers and attended upper-middle-class public schools. The adults were 32 psychology students at the Université de Bourgogne (29 female and 3 male, mean age $=22 ; 8$ years, $S D=47.8$ months).

Material and procedure. Round or square dots, either red or yellow, were arranged randomly on six $29.7 \times 42$-cm sheets for the small arrays ( 8 to 13 targets) and six $29.7 \times 84-\mathrm{cm}$ sheets for the large arrays (20 to 25 targets). For both sizes, half of the arrays corresponded to the one-feature condition and the other half to the two-feature condition. In the former, the targets were red (circles or squares), and in the latter, they were red circles. The number of distracters (i.e., yellow circles and squares in the one-feature condition, red squares and yellow circles and squares in the two-feature condition) was one-third greater than the number of targets. 
The procedure was similar to those of Experiments $2 \mathrm{a}$ and $2 \mathrm{~b}$. In the saying task, the participants were asked to count aloud from 0 to 30 . We recorded the saying times from 0 to 13 and from 0 to 25 , and the number of errors. To shorten the experiment, the children performed the task just once. The pointing and counting tasks were identical to those used in Experiments $2 \mathrm{a}$ and $2 \mathrm{~b}$. For each of the two tasks, half of the participants pointed and counted the one-feature and then the two-feature arrays and the other half in the reverse order.

\section{Results}

Because more than $75 \%$ of the 6-year-old children did not achieve at least one correct trial in each of the three tasks (saying, pointing, and counting) when large arrays were presented, the following analyses were computed on the small arrays only. As in the previous experiments, we analyzed the times for the successful trials only, thus retaining 19 6-year-old children, 28 9-year-old children, and 32 adults (Table 4).

As we expected, pointing and counting were slowed down in the two-feature condition compared to the one-feature condition. Pointing in the two-feature condition took longer $(408 \mathrm{~ms})$ than in the one-feature condition $(380 \mathrm{~ms}), F(1,76)=$ $43.3, p<.001, M S_{\mathrm{e}}=1062$, and resulted in more errors $(23$ vs $13 \%), F(1,84)=$ $11.1, p<.01, M S_{\mathrm{e}}=423$. The Age effect was significant (6-year-old: $603 \mathrm{~ms}$ and $33 \%$ errors, 9-year-old: $418 \mathrm{~ms}$ and $21 \%$, and adults: $249 \mathrm{~ms}$ and $4 \%), F(2,76)=$ $186.6, p<.001, M S_{\mathrm{e}}=8103$, for the times, and $F(2,84)=17.7, p<.001, M S_{\mathrm{e}}=$ 686 , for the errors, and interacted with the type of target. The younger participants

TABLE 4

Mean Times (ms) and Percentage of Errors (in Parentheses) in Experiment 3

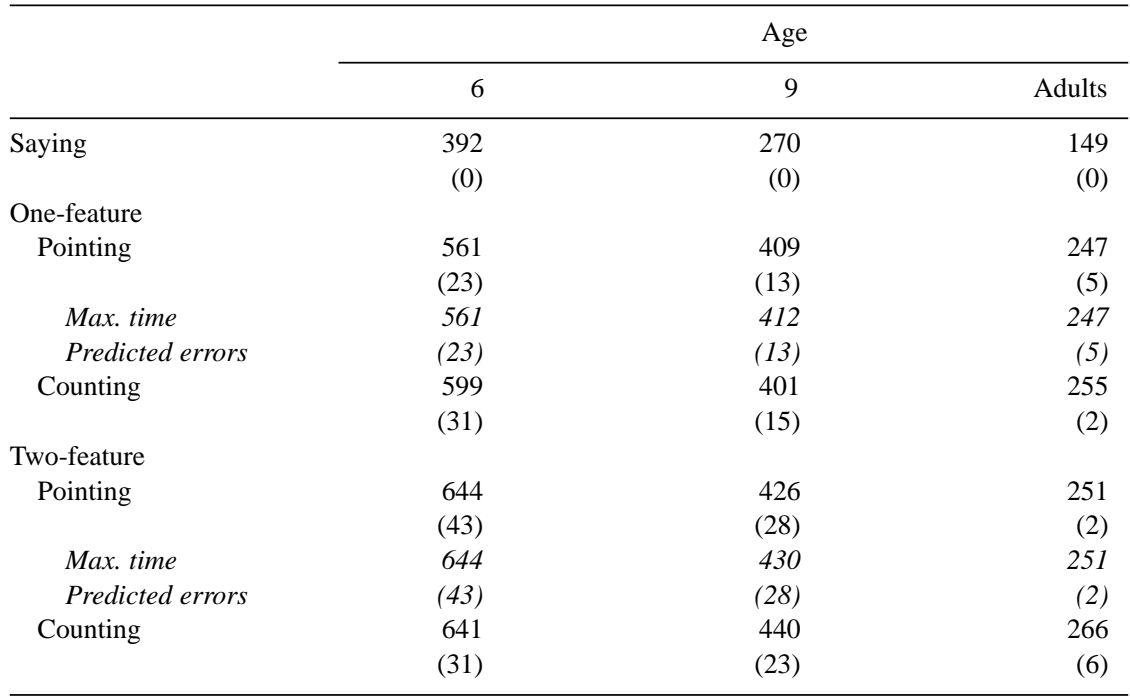


were more affected by the type of target (644 ms and $43 \%$ errors for two-feature vs $561 \mathrm{~ms}$ and $23 \%$ for one-feature condition) compared to the 9-year-old children (426 ms and $28 \%$ vs $409 \mathrm{~ms}$ and $13 \%$, respectively) and the adults (251 ms and $2 \%$ vs $247 \mathrm{~ms}$ and $5 \%), F(2,76)=18.8, p<.001, M S_{\mathrm{e}}=1062$, for the times, and $F(2,84)=5.05, p<.01, M S_{\mathrm{e}}=423$, for the errors.

The effects on counting were approximately identical. Counting was slower in the two-feature condition $(418 \mathrm{~ms})$ than in the one-feature condition $(390 \mathrm{~ms})$, $F(1,76)=30.2, p<.001, M S_{\mathrm{e}}=1133$, but it did not result in a higher error level (19 vs $15 \%$, respectively). The Target effect interacted with age only for times, $F(2,76)=3.7, p<.05, M S_{\mathrm{e}}=1133$, but not for the errors, $F<1$. Indeed, the target effect was smaller in adults (266 vs $255 \mathrm{~ms}$ for two- and one-feature conditions, respectively) than in 6-year-old (641 vs $599 \mathrm{~ms}$ ) and 9-year-old (440 vs 401 $\mathrm{ms}$ ) children. The type of target had no significant effect on error rates, which diminished with age ( $31 \%$ at $6,19 \%$ at 9 , and $4 \%$ among adults), $F(2,84)=12.8$, $p<.001, M S_{\mathrm{e}}=792$. These results bore out our expectations. Because they are more difficult to point to, the two-feature targets led to slower counting.

The hypothesis of a costly coordination between pointing and saying predicted that the differences between (a) $C$ and Max $[P, S]$ times, and (b) the observed and the predicted error rates should be all the higher the more difficult the pointing was (i.e., in the two-feature condition). A $3($ Age $) \times 2$ (Time: counting vs Max $[P, S]) \times$ 2 (Target: one vs two features) ANOVA with repeated measures on the two last factors was performed on the times. Contrary to the costly coordination hypothesis, there was no significant interaction between time and target, $F<1$. Furthermore, this interaction interacted with age, $F(2,76)=8.9, p<.001, M S_{\mathrm{e}}=672$, but, once more, in the opposite direction from the one predicted. Indeed, the difference between $C$ and Max was larger in the two-feature than in the one-feature condition in adults (15 and $8 \mathrm{~ms}$, respectively) and 9-year-old children (10 and $-11 \mathrm{~ms})$, but this effect was reversed in 6-year-old children ( -3 vs $38 \mathrm{~ms}$ for two and one feature, respectively). The same phenomenon was observed in error rates. There was no interaction between rates (observed vs predicted) and target, $F<1$, and the Age $\times$ Rate $\times$ Target interaction was significant and showed the same pattern, $F(2,84)=3.8$, $p<.05, M S_{\mathrm{e}}=345$. As observed in times, the difference between observed and predicted error rates increased with the difficulty of pointing in adults ( -3 vs $4 \%$ for one and two features, respectively), but decreased in 9-year-old children ( 2 and $-5 \%)$ and much more in 6 -year-old children ( 8 and $-12 \%)$.

In summary, although the constraints affecting pointing had the expected effect in both the pointing and the counting tasks, their effect on the difference between (a) the counting and the $\operatorname{Max}[P, S]$ times, and (b) the observed and predicted rates of errors was not compatible with the hypothesis that counting requires a demanding coordination between pointing and saying.

\section{GENERAL DISCUSSION}

These experiments compared (a) the time to count an array with the time to perform the slowest component, either pointing or saying, and (b) the rate of 
errors observed in counting with the rate predicted by the independence of the two components. Assuming that the coordination of pointing and saying is demanding, it should result in longer times and higher error rates when participants count than when they point or say the number line. This additional time requirement and increase in errors should vary depending on the participant's age and on the difficulty experienced by the participant in performing one or other of the two components. The results obtained do not support this hypothesis, and reinforce the results of Towse and Hitch (1997) and Miller and Stigler (1987). Counting is frequently faster than the slowest of its components without any notable increase in errors (Experiments 1 and 3 for the youngest children, Experiment $2 \mathrm{~b}$ with English and Tahitian in adults). While saying, pointing, and counting speeds increase with age, the difference in times between counting and the slowest component tends to increase, not decrease. This difference remains unaffected by any variations in the difficulty of saying (Experiments $2 a$ and $2 b$ ) or pointing (Exp. 3) which equally affect counting speed. As observed by Towse and Hitch (1997) or Miller and Stigler (1987), the age and the difficulty of pointing or saying affect counting speed, but not the coordination.

Counting therefore is a complex activity in which efficiency, evaluated by speed and accuracy, is affected by any factor liable to hinder the progress of its components: pointing and saying. However, our results, like those of Towse and Hitch (1997), suggest that the integration of the two components in counting presents a negligible cognitive cost, even at age 5. Note that, unlike the dual-task situations in which the interactions expected by Towse and Hitch (1997) are frequently observed (Barrouillet \& Fayol, 1998; Anderson et al., 1996), counting is an integrated activity in which pointing and saying converge toward the same goal and must progress together. This makes counting a particularly suitable task for the study of coordination. It is also a basic skill which benefits from early learning and which is used frequently in daily life. It is therefore difficult to imagine that the integration of pointing and saying (i.e., management of their synchrony) requires extensive attentional control, which would make counting more costintensive and less reliable.

The observed results are more compatible with the hypothesis that, after age 5, counting is what Anderson (1993) calls a procedure (or a production rule). Anderson supposes that there are two types of knowledge: declarative and procedural. This dichotomy is necessary because the cognitive system requires different knowledge depending on the way it is used, either flexible and not committed to a particular use (declarative knowledge), or limited to specific situations but efficient (procedural knowledge). Procedures optimize the use of knowledge, such as the number line, in a specific application (e.g., counting). Procedures are goal-directed (for example, determining the cardinal corresponding to an array), and modular; i.e., they constitute the units of the skills. According to Anderson, these production rules result from a compilation of a series of actions. When the production rule is formed, it performs all the information processing necessary to attain the goal: "a production rule is a compiled procedure [ . . . that requires the setting up of its 
own control structure" (Anderson, 1993, p. 86). Within this concept, the establishment of procedural knowledge would solve problems due to information management and to its processing during frequently repeated operations.

We can suppose that the operations necessary to count an array (pointing and saying) are proceduralized at an early age by means of a compilation process, which enables their integrated mobilization without the need for attentional control of the coordination. The procedure is responsible for the mobilization of the various actions in the sequence (identifying the next item, pointing at it, retrieving the corresponding word for articulation) as well as for controlling their sequencing and coordination. Thus, the difficulty in identifying the objects to be counted, or the use of a less automatized verbal chain than the number line, would slow down a given processing step, and therefore the entire process, without increasing the demand of coordination. When pointing alone is required, the retrieval and production of the verbal item would be eliminated thus resulting in shorter time requirements than counting. Indeed, in most of the previous experiments, counting was slower than pointing. As might be assumed, in young children the retrieval of the verbal items is slower for a less automatized chain (e.g., alphabet vs numbers) and therefore counting with the alphabet takes more time than counting with numbers. The same phenomenon is observed when adults have to count in a foreign language. Similarly, factors that affect pointing speed consequently also affect counting speed. However, these factors do not affect the retrieval process and the production of the number-word. Thus, in Experiment 3, the difference between counting and the slowest component was not sensitive to the discrimination of targets. In Towse and Hitch's experiments, variations in the difficulty of locating the objects and in saying the appropriate word had the same effects on counting times. However, these effects are additive because the role of the proceduralization of the activity is to ensure the integration of the various components which then no longer require attentional control or any specific resource allocation.

Thus, the development of counting in children at least after 5 years of age, cannot be accounted for by the decreasing cognitive load of the integration of verbal and motor information by means of a coordination process monitored by a central executive. Instead, counting is a procedure that is reinforced by practice (Anderson, 1993; Anderson, Finchman, \& Douglass, 1997), resulting in the agerelated speed increase observed in our experiments. This practice-related effect could be reinforced by a general increase in processing speed which Kail (1991) considers to be one of the determining factors of cognitive development.

Finally, let us return to one of our most provocative results, that is, the facilitation effect for young children when they had to perform pointing and saying simultaneously rather than separately (Experiment 1). Similar phenomena have been described by Yee, Hunt, and Pellegrino (1991). These authors subjected their participants to a perceptual task and a verbal task which were performed either separately or simultaneously. They observed that (a) the coordination of easy tasks induced an additional time requirement greater than that necessary to coordinate 
difficult tasks (compression effect), and (b) under certain conditions, simultaneous completion took less time than separate completion (e.g., Experiment 4). Accordingly, we observed that counting times were frequently shorter than the times of the slowest component (often saying) in younger children, although the opposite was the case in adults. It is interesting to note that when the adults were asked to count in a foreign language, their counting performance was close to that of young children, ${ }^{1}$ and resulted in counting times shorter than times of the slowest component (see Case, Kurland, \& Goldberg, 1982, for similar observations). In both cases it appears that when the number line is embedded within a counting procedure, its production is facilitated and accelerated.

This phenomenon reflects Luria's observations $(1959,1961)$ that verbal triggers (saying "go" while simultaneously pressing a button in response to a light signal) supplement, in young children, the still inadequate control of their motor activity. We have observed that dyspraxic children were able to count arrays rapidly and with few errors although these arrays were difficult for them to point at (Camos, Fayol, Lacert, Bardi, \& Laquière, 1998). Conversely, it is possible that, because the activities in the counting procedure are integrated, the motor activity of pointing plays a release role in the retrieval of the successive verbal items, at least while the employed verbal chain is not fully automatized. This might explain why it is mainly the young children and the adults counting in a foreign language who exhibit counting times shorter than the times of the slowest component. Murphy and Peters (1994) have observed identical phenomena in reading: the addition of a concurrent tapping task to a word reading task resulted in an increase in the number of words read.

Although being frequently observed, these facilitation effects, which contradict all the main cognitive models, still need explanatory hypotheses and further research. The classical approach which conceives the development of complex skills as a progressive integration of previously independent components (Case, 1985; Piaget, 1959) are not well suited to the understanding of early and highly practiced activities.

When the verbal chain is automatized (e.g., in adults in their native language), counting times are rarely shorter than the times of the slowest component (generally pointing). This fact suggests that, even in adults, saying and pointing cannot be performed totally in parallel, and that part of the processing could remain sequential. On the one hand, the completion of a step (e.g., reaching the object to point) could constitute one of the release conditions for the next step (i.e., saying the word retrieved from memory). On the other hand, although the two components can usually be completed in parallel, certain common treatments (e.g., the selection of the verbal and motor responses) could involve a common process resulting in a bottleneck (Pashler, 1993, 1994a, 1994b) or output interference (Brainerd \& Reyna, 1989).

${ }^{1}$ In Experiment 1, 5-year-old children had a mean counting time of $570 \mathrm{~ms}$ and a mean error rate of $66 \%$, whereas adults in Experiment $2 \mathrm{~b}$ had a mean counting time of $655 \mathrm{~ms}$ and an error rate of $51 \%$ when they counted in Tahitian. 
To conclude, counting is a procedure, at least from age 5. However, this procedure is efficient at an early age, it involves little cost and shows little potential for development. Thus, the development of counting might also consist of a change of the strategies used, as in other domains of arithmetic knowledge (Barrouillet \& Fayol, 1998; Siegler, 1996). Pointing with a finger might be replaced in adults by a visual tagging of the targets, which would allow iterative additions of the outcomes of successive subitizings. The factors constraining this strategy, probably the most frequent in adults, differ from those affecting counting one by one with motor pointing, favored by young children. Counting in adults is constrained by the retrieval of additive facts from memory and the relative ease of isolating subgroups of objects that can be subitized. Thus, the development of counting may be more the result of the acquisition of arithmetic knowledge than of a decrease in the cognitive cost of the primitive strategy.

\section{REFERENCES}

Anderson, J. R. (1993). The rules of the mind. Hillsdale; NJ: Erlbaum.

Anderson, J. R., Finchman, J. M., \& Douglass, S. (1997). The role of examples and rules in the acquisition of a cognitive skill. Journal of Experimental Psychology: Learning, Memory, and Cognition, 23, 932-945.

Anderson, J. R., Reder L. M., \& Lebière, C. (1996). Working memory: Activation limitations on retrieval. Cognitive Psychology, 30, 221-256.

Aoki, T. (1977). On the counting process of patterned dots. Tohoku Psychologica Folia, 36, 15-22.

Baddeley, A. D. (1990). Human memory, Theory and practice. Hillsdale, NJ: Erlbaum.

Baddeley, A. D., \& Hitch, G. J. (1974). Working memory. In G. A. Bower (Ed.), Recent advances in learning and motivation (Vol. 8). New York: Academic Press.

Barrouillet, P., \& Fayol, M. (1998). From algorithmic computing to direct retrieval : Evidence from number and alphabetic arithmetic in children and adults. Memory \& Cognition, 26, 355-368.

Beckwith, M., \& Restle, F. (1966). Process of enumeration. Psychological Review, 73, 437-444.

Brainerd, C. J., \& Reyna, V. F. (1989). Output-interference theory of dual-task deficits in memory development. Journal of Experimental Psychology, 47, 1-18.

Briars, D., \& Siegler, R. S. (1984). A feature analysis of preschoolers' counting knowledge. Developmental Psychology, 20, 607-618.

Camos, V., Fayol, M., Lacert, P., Bardi, A., \& Laquière, C. (1998). Le dénombrement chez cinq enfants dysphasiques et cinq enfants dyspraxiques. Approches Neuropsychologiques des Apprentissages chez l'Enfant, 48, 86-91.

Case, R. (1985). Intellectual development: Birth to adulthood. New York: Academic Press.

Case, R., Kurland, M., \& Goldberg, J. (1982). Operational efficiency and the growth of short-term memory span. Journal of Experimental Child Psychology, 33, 386-404.

Crannel, C. W., \& Parrish, J. M. (1957). A comparison of immediate memory span for digits, letters, and words. The Journal of Psychology, 44, 319-327.

Dehaene, S., \& Mehler, J. (1992). Cross-linguistic regularities in the frequency of number words. Cognition, 43, 1-29.

Dempster, F. N. (1981). Memory span: Sources of individual and developmental differences. Psychological Bulletin, 89, 63-100.

Fayol, M. (1985). Nombre, numération et dénombrement: que sait-on de leur acquisition? Revue Française de Pédagogie, 70, 59-77.

Fayol, M. (1990). L'enfant et le nombre. Paris: Delachaux et Niestlé.

Fayol, M., Gombert, J. E., Lecocq, P., Sprenger-Charolles, L., \& Zagar, D. (1992). La psychologie cognitive de la lecture. Paris: Presses Universitaires de France. 
Frick, R. W. (1987). The homogeneity effect in counting. Perception \& Psychophysics, 41, 8-16.

Fuson, K. C. (1988). Children's counting and concepts of number. New York: Springer-Verlag.

Fuson, K. C., Richards, J., \& Briars, D. J. (1982). The acquisition and elaboration of number word sequence. In C. Brainerd (Ed.), Progress in cognitive development, Vol. 1, Children's logical and mathematical cognition. New York: Springer-Verlag.

Gelman, R. (1983). Les bébés et le calcul. La Recherche, 14, 1382-1389.

Gelman, R., \& Gallistel, C. R. (1978). The child's understanding of number. Cambridge, MA: Harvard Univ. Press.

Gelman, R., \& Meck, E. (1983). Preschooler's counting: Principles before skills. Cognition, 13, 343-359.

Gréco, P. (1962). Quantité et quotité. In P. Gréco \& A. Morf (Eds.), Structures numériques élémentaires (Etudes d'épistémologie génétique, Vol. XIII) (pp. 1-70). Paris: P.U.F.

Greeno, J. G., Riley, M. S., \& Gelman, R. (1984) Conceptual competence and children's counting. Cognitive Development, 16, 94-143.

Halford, G. S. (1993). Children's understanding: The development of mental models. Hillsdale, NJ: Erlbaum.

Kail, R. (1991). Developmental change in speed of processing during childhood and adolescence. Psychological Bulletin, 109, 490-501.

Logan, G. D., \& Klapp, S. T. (1991). Automatizing alphabet arithmetic: I. Is extended practice necessary to produce automaticity? Journal of Experimental Psychology: Learning, Memory and Cognition, 17, 179-195.

Logie, R. H., \& Baddeley, A. D. (1987). Cognitive processes in counting. Journal of Experimental Psychology: Learning, Memory and Cognition, 13, 310-326.

Luria, A. R. (1959). The directive function of speech in development and dissolution. Part I: Development of the directive function of speech in early childhood. Word, 15, 341-352.

Luria, A. R. (1961). The role of regulation of speech in the regulation of normal and abnormal behaviour. New York: Pergamon Press.

Miller, K. F. (1996). Origins of quantitative competence. In Perceptual and cognitive development (pp. 213-241). New York: Academic Press.

Miller, K. F., \& Stigler, J. W. (1987). Counting in Chinese: Cultural variation in a basic cognitive skill. Cognitive Development, 2, 279-305.

Miura, I. T., Okamoto, Y., Kim, C. C., Chang, C. M., Steere, M., \& Fayol, M. (1994). Comparisons of children's cognitive representation of number: China, France, Japan, Korea, Sweden and the United States. International Journal of Behavioral Development, 17, 401-411.

Murphy, K., \& Peters, M. (1994). Right-handers and left-handers show differences and important similarities in task integration when performing manual and vocal tasks concurrently. Neuropsychologia, 32, 663-674.

Nairne, J. S., \& Healy, A. F. (1983). Counting backwards produces systematic errors. Journal of Experimental Psychology: General, 112, 37-40.

Navon, D. (1984). Resources-A theoretical soup stone? Psychological Review, 91, 216-234.

Newman, R. S., Friedman, C. A., \& Gockley, D. R. (1987). Children's use of multiple-counting skills: Adaptation of task factors. Journal of Experimental Child Psychology, 44, 268-282.

Pascual Leone, J. (1988). Organismic processes for neo-piagetian theories: A dialectical causal account of cognitive development. In A. Demetriou (Ed.), The neo-piagetian theories of cognitive development: Toward an integration (pp. 25-64). Amsterdam: North Holland.

Pashler, H. (1993). Doing two things at the same time. American Scientist, 81, 48-55.

Pashler, H. (1994a). Graded capacity-sharing in dual-task interference? Journal of Experimental Psychology: Human Perception and Performance, 20, 1-13.

Pashler, H. (1994b). Dual-task interference in simple tasks: Data and theory. Psychological Bulletin, 116, 220-244.

Piaget, J. (1959). La naissance de l'intelligence chez l'enfant. Neuchâtel: Delachaux \& Niestlé.

Piaget, J., \& Szeminska, A. (1941). La genèse du nombre chez l'enfant Neuchâtel: Delachaux \& Niestlé. 
Potter, M. C., \& Levy, E. I. (1968). Spatial enumeration without counting. Child Development, 39, $265-272$.

Schaeffer, B., Eggleston, V., \& Scott, J. L. (1974). Number development in young children. Cognitive Psychology, 6, 357-379.

Shannon, L. (1978). Spatial strategies in the counting of young children. Child Development, 49, 1212-1215.

Siegler, R. S. (1996). Emerging minds: The process of change in children's thinking. Oxford: Oxford Univ. Press.

Sternberg, S. (1969). The discovery of processing stages: Extensions of Donders' method. Acta Psychologica, 30, 276-315.

Towse, J. N., \& Hitch, G. J. (1996). Performance demands in the selection of objects for counting. Journal of Experimental Child Psychology, 61, 67-79.

Towse, J. N., \& Hitch, G. N. (1997). Integrating information in object counting: A role for a central coordination process?. Cognitive Development, 12, 393-422.

Treisman, A., \& Gelade, G. (1980). A feature-integration theory of attention. Cognitive Psychology, 12, 97-136.

Trick, L. M., \& Pylyshyn, Z. W. (1994). Why are small and large numbers enumerated differently? A limited-capacity preattentive stage in vision. Psychological Review, 101, 80-102.

Turner, M. L., \& Engle, R. W. (1989). Is working memory task dependent? Journal of Memory and Language, 28, 127-154.

Wilkinson, A. C. (1984). Children's partial knowledge of the cognitive skill of counting. Cognitive Psychology, 16, 28-64.

Yee, P. L., Hunt, E., \& Pellegrino, J. W. (1991). Coordinating cognitive information: Task effects and individual differences in integrating information from several sources. Cognitive Development, 23, 615-680.

Received June 8, 1998; revised February 25, 2000 